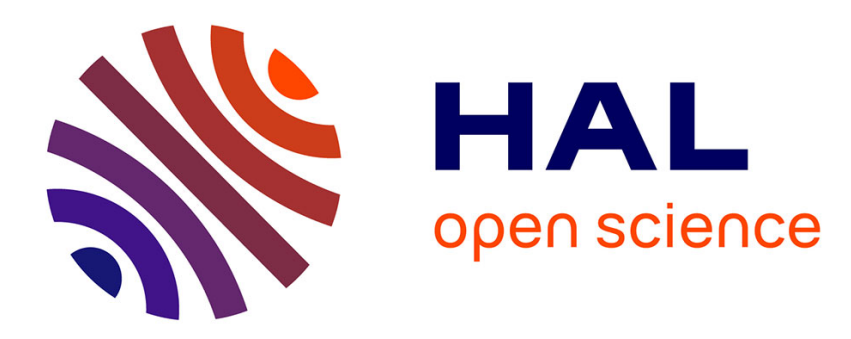

\title{
Les émeutes dans la sociologie américaine
}

François Bonnet

\section{To cite this version:}

François Bonnet. Les émeutes dans la sociologie américaine. Mouvements: des idées et des luttes, 2015, 83, pp.138-144. halshs-01199612

\section{HAL Id: halshs-01199612 https://shs.hal.science/halshs-01199612}

Submitted on 15 Sep 2015

HAL is a multi-disciplinary open access archive for the deposit and dissemination of scientific research documents, whether they are published or not. The documents may come from teaching and research institutions in France or abroad, or from public or private research centers.
L'archive ouverte pluridisciplinaire HAL, est destinée au dépôt et à la diffusion de documents scientifiques de niveau recherche, publiés ou non, émanant des établissements d'enseignement et de recherche français ou étrangers, des laboratoires publics ou privés. 
Les émeutes dans la sociologie américaine

François Bonnet

CNRS, UMR Pacte

Version 2, 21 mai 2015

Les Etats-Unis ont connu des émeutes massives et meurtrières dans les années 1960, et des épisodes plus dispersés après. Cet article rend compte de la littérature américaine sur les émeutes, et notamment du progressif désintérêt des sociologues américains pour leur dimension protestataire, au profit d'analyses formalistes. Cette dimension a pourtant été mise en lumière par les événements de Ferguson en 2014 et Baltimore en 2015, qui sont venus rappeler que les ghettos noirs se soulèvent d'abord, sinon seulement, en réaction à l'intense répression policière qui s'y déploie.

\begin{abstract}
«S'il n'y avait pas eu de soulèvement général des ghettos noirs à travers le pays, les leaders noirs auraient pu se faire tuer sans l'ombre d'une hésitation par n'importe quel blanc raciste... Etait-il concevable que nous laissions passer la mort de Dr. [Martin Luther] King sans aucune forme de représailles?»

-- Révérend Albert B. Cleage, Jr., Detroit ${ }^{1}$
\end{abstract}

Au cours des années 1960, les Etats-Unis connaissent une série d'émeutes dans les ghettos noirs qui font près de 200 morts. Ecartons tout de suite les questions de vocabulaire : la sociologie américaine emploie une multitude de termes : émeute, émeute urbaine, émeute raciale, violence urbaine, rébellion, insurrection, désordre, dérangement (disturbance), soulèvement, protestation, événement... La sociologie américaine ne dispose pas non plus d'une définition satisfaisante des émeutes, ni ne sait comment se dépêtrer de l'ambivalence entre leur dimension protestataire, et leur aspect de simple violence plus ou moins criminelle. Cet article présente donc la sociologie américaine des émeutes, qui suit une trajectoire intrigante. La sociologie des années 1960, en réaction contre les interprétations conservatrices du débat public, est pleine de sympathie pour les émeutiers noirs. Puis, dans les années 1970, Seymour Spilerman publie une série d'articles qui montrent que les indicateurs de pauvreté ne sont pas corrélés aux émeutes. La sociologie américaine des émeutes devient alors une discipline technique, portée sur la prouesse méthodologique, sourde et aveugle à leur signification sociale et politique. Les événements de Ferguson et Baltimore jettent une lumière crue sur cette dérive technicienne.

\title{
Les émeutes des années 1960
}

Entre 1964 et 1968, on recense 239 émeutes dans 215 villes, pour près de 50000 personnes arrêtées, 8000 blessés et 191 morts. Dans les quartiers concernés, les émeutes sont populaires : les enquêtes montrent de façon concordantes que la moitié des habitants (noirs) soutiennent les émeutiers, et qu'environ $15 \%$ des habitants du quartier ont participé d'une façon ou d'une autre ${ }^{2}$. Ces émeutes scandalisent et terrifient la plupart des Américains (blancs). Les slogans de l'émeute de Los Angeles de 1965 sont «burn, baby, burn » et «get

\footnotetext{
${ }^{1}$ Cité in Drake, St. C. (1968). Urban violence and American social movements. Proceedings of the Academy of Political Science, 29 (1), p. 23

${ }^{2}$ Sears, D. O., \& McConahay, J. B. (1969). Participation in the Los Angeles riot. Social Problems, 17(1), p. 8
} 
Whitey !»-Whitey est un terme péjoratif pour désigner les blancs. Les émeutes impliquent des incendies, des pillages, et le lynchage d'automobilistes blancs malheureux qui passaient au mauvais endroit au mauvais moment. Après l'abolition de l'esclavage en 1865, les Noirs étaient restés concentrés dans le sud, mais les deux guerres mondiales et la croissance d'aprèsguerre avaient provoqué un besoin de main d'œuvre qui avait attiré des millions de Noirs vers les grandes villes industrielles. Le racisme et la ségrégation avaient conduit les Noirs à s'entasser dans les ghettos de ces villes. A Chicago, entre 1910 et 1970, la proportion de la population noire passe de 2 à $33 \%$. Les blancs des classes populaires - souvent juifs, italiens ou irlandais - voient leur quartier changer, la valeur de l'immobilier s'effondrer, et les Noirs piller et incendier les villes.

Dans les termes du débat public, les émeutes sont largement commises par le riffraff (la racaille, la lie) de la société (noire): les fainéants, bons-à-riens et criminels qui instrumentalisent à bon compte le mouvement des droits civiques pour piller des magasins. Le chef Parker, du Los Angeles Police Department (LAPD), décrit les émeutiers comme «des singes dans un zoo ». Certains analysent les émeutes comme le résultat d'une conspiration des Noirs pour renverser l'ordre établi, et mentionnent la fabrication étrangement professionnelle de cocktails Molotov, ou les coups de téléphone suspects qui guident la police dans la ligne de mire d'un sniper. Les policiers de Cambridge (Maryland) vont par exemple empêcher les pompiers d'éteindre un incendie dans le ghetto, et le laisser s'étendre, car ils craignent une ruse pour éloigner les pompiers du quartier d'affaires.

Dans ce contexte, les sociologues s'attachent à montrer que les émeutes sont un mouvement social contre la pauvreté et l'oppression. Le schéma explicatif est inspiré de Neil Smelser, le grand sociologue de Berkeley. Le comportement violent est conçu comme résultant d'une frustration, par exemple due à la pauvreté (deprivation-frustration-aggression hypothesis) : les contraintes structurelles favorisent le ressentiment et la colère ; un incident déclencheur permet la mobilisation; la réaction des autorités pacifie ou aggrave la situation ${ }^{1}$. Robert Fogelson, futur historien au MIT, analyse les émeutes comme «des tentatives d'attirer l'attention de la société blanche sur l'insatisfaction généralisée des [Noirs] envers la subordination raciale et la ségrégation ${ }^{2}$ ». Herbert Gans, futur professeur à Columbia, parle de « rébellions » qui «sont le résultat naturel d'années de colère » ${ }^{3}$. Contre la théorie du riffraff, Caplan décrit l'émeutier comme "the new ghetto man»: un militant déterminé, éduqué, conscientisé, loin des stéréotypes du Noir du sud résigné et analphabète ${ }^{4}$. St. Clair Drake (l'auteur de Black Metropolis) rappelle que les abolitionnistes (de l'esclavage), les suffragettes et le mouvement ouvrier ont tous été taxés «d'émeutiers » 5 .

La plupart des émeutes des années 1960 sont analysées comme «raciales » au sens où elles sont menées par des Noirs dans le contexte du mouvement pour les droits civiques, au contraire des émeutes des XIXe et début du XXe siècle, qui étaient des émeutes « communales » (selon le terme de Morris Janowitz), c'est-à-dire opposant des habitants se révoltant contre l'arrivée d'immigrés dans leur quartier. Dans les faits, certaines des émeutes des années 1960 sont articulées à un mouvement de revendications, d'autres semblent

\footnotetext{
${ }^{1}$ McPhail, C. (1994). The dark side of purpose. Sociological Quarterly, 35(1), p. 3

${ }^{2}$ Fogelson, R. M. (1968). Violence as protest. Proceedings of the Academy of Political Science, 29 (1), p. 37-38.

${ }^{3}$ Gans, H. J. (1968). The ghetto rebellions and urban class conflict. Proceedings of the Academy of Political Science, 29 (1), p. 42.

${ }^{4}$ Caplan, N. (1970). The new ghetto man: A review of recent empirical studies. Journal of Social Issues, 26(1), 59-73.

${ }^{5}$ Drake, St. C., op. cit.
} 
exclusivement orientées vers le pillage, et des bagarres entre ivrognes à la sortie des boites de nuit ont été étiquetées "émeutes» parce que des Noirs étaient impliqués. L'émeute de Chicago 1968 lors de la convention du Parti démocrate est une émeute policière : les policiers déchaînés se sont laissés aller à la violence collective. Pour les sociologues des années 1960, il est clair que les émeutiers des ghettos noirs se révoltent contre l'injustice raciale. Cette perspective triomphe dans le rapport Kerner, commandé par le président Lyndon Johnson à une commission présidée par le gouverneur de l'Illinois, Otto Kerner. Publié en 1968, le rapport souligne le rôle joué par la pauvreté et le racisme et énonce : «notre pays se dirige vers deux sociétés, l'une noire, l'autre blanche - séparées et inégales ». Les recommandations du rapport Kerner apparaissent trop radicales et sont rapidement mises de côté. Un mois après la publication, Martin Luther King est assassiné, ce qui déclenche une importante vague d'émeutes à travers le pays.

\section{Le tournant Spilerman}

Quelles sont les causes profondes des émeutes raciales des années 1960 ? Le sociologue Seymour Spilerman compare les 673 villes de plus de 25000 habitants entre celles qui ont connu des émeutes et les autres ; pour chacune des villes, il regarde de nombreuses variables liées la pauvreté, aux inégalités, et à la « désorganisation sociale », comme le pourcentage de Noirs au chômage, les taux de criminalité, les inégalités de revenus entre Blancs et Noirs. Son analyse statistique sophistiquée produit des résultats contre-intuitifs : les émeutes ne sont corrélées à aucune variable socio-économique. Seule la taille de la ville importe : plus une ville est grande, plus elle a de chances de connaître une émeute ${ }^{1}$. Puis Spilerman, dans un autre article, analyse l'intensité des émeutes, avec la même idée : expliquer pourquoi une émeute s'aggrave, en se focalisant sur les variables socio-démographiques. Même conclusion : aucune des variables liées à la pauvreté ou aux inégalités n'est corrélée à la l'intensité des émeutes. Spilerman spécule en conclusion que la télévision est probablement « le mécanisme essentiel de la contagion des émeutes ${ }^{2} »$.

Les résultats de Spilerman ont été répliqués et confirmés vingt ans plus tard. Ces résultats sont paradoxaux : si les émeutes n'ont rien à voir avec la pauvreté, pourquoi ont-elles toutes lieu dans des ghettos noirs pauvres ? Une possibilité est qu'il n'y a pas de corrélation linéaire entre « causes sociales » et émeutes, mais un effet de seuil. Si la très grande majorité des Noirs sont plus pauvres que les blancs et victimes de racisme, il n'est pas illogique que dans toutes les villes, ils soient au dessus d'un seuil de propension à l'émeute. Dans cette perspective, les variations en-dessous de ce seuil ne sont pas significatives; la présence ou non d'émeutes dépend de la contingence de l'incident déclencheur.

Peu importe: Spilerman a gagné. Après Spilerman, dans les années 1980 et 1990, la sociologie des émeutes devient une entreprise technique, formaliste, qui cherche à formuler des modèles épidémiologiques, situationnels, voire cybernétiques. Un politiste modélise le problème du passager clandestin chez les émeutiers : il devient rationnel de piller quand il y a suffisamment de monde autour de soi pour réduire la probabilité d'arrestation. Les travaux sur la violence gratuite et nihiliste des hooligans sont pris comme modèle du comportement émeutier ${ }^{3}$. Bert Useem propose d'analyser les émeutes non comme des mouvements sociaux,

\footnotetext{
${ }^{1}$ Spilerman, S. (1970). The causes of racial disturbances: A comparison of alternative explanations. American Sociological Review, 35 (4): 627-649.

${ }^{2}$ Spilerman, S. (1976). Structural characteristics of cities and the severity of racial disorders. American Sociological Review, 41 (5): p. 790.

${ }^{3}$ Voir la revue de littérature de McPhail, C., \& Wohlstein, R. T. (1983). Individual and collective behaviors within gatherings, demonstrations, and riots. Annual Review of Sociology, 9: 579-600.
} 
mais comme des effondrements des normes sociales (social breakdown) ${ }^{1}$. Susan Olzak, professeure à Stanford, affine l'analyse de Spilerman en suggérant que la compétition (pour l'espace et pour les opportunités économiques), notamment après une période de ségrégation stricte, favorise les émeutes ${ }^{2}$. Ce schéma semble adapté pour les principales émeutes des années 1980-1990 : Miami (1980, 17 morts), Los Angeles (1992, 52 morts) et Crown Heights (1994, un mort). Ces émeutes ont une dimension «communale»: à Miami, les Noirs sont fragilisés sur le marché du travail par l'arrivée massive d'immigrés cubains et haïtiens ${ }^{3}$. A Los Angeles, les ghettos noirs sont progressivement transformés par l'arrivée d'immigrés asiatiques et latino, et les supérettes coréennes sont les cibles principales des émeutiers ${ }^{4}$. A Crown Heights, ce sont les conflits entre Noirs et nouveaux arrivants juifs hassidiques qui sont en cause. Dans les grandes revues comme l'American Sociological Review ou l'American Journal of Sociology, le prisme de l'émeute comme protestation contre l'injustice sociale et raciale est enterré. L'émeute de Cincinnati de 2001 ne fait même pas de morts ; les émeutes sortent du débat public et scientifique aux Etats-Unis.

\section{Le retour de l'histoire}

En 2008, le grand historien progressiste Michael Katz (The Undeserving Poor) publie un article intitulé : "pourquoi est-ce que les villes américaines ne brûlent pas très souvent?». L'étonnement provient de la comparaison avec les émeutes françaises de 2005, mentionnées dans la première phrase de l'article. Pourquoi est-ce que les villes françaises brûlent, mais pas les villes américaines ? Katz identifie trois raisons : l'hyperségrégation américaine qui limite les contacts et donc les conflits raciaux, l'émergence d'une classe moyenne noire et la cooptation des élites noires protestataires (dans la grande tradition du colonialisme britannique), et la meilleure intégration économique et politique des immigrés américains ${ }^{5}$. Les émeutes de Villiers-le-Bel (2007), Firminy (2009), Grenoble (2010), Amiens (2012) et Trappes (2013) donnent raison à Katz : les banlieues françaises brûlent, tandis que les ghettos américains seulement sont stables. On pense arriver à la « fin de l'histoire » des émeutes aux Etats-Unis, doublement enterrées comme objet d'intérêt sociologique par Spilerman puis par la disparation des émeutes.

C'est dans ce contexte que se succèdent, en quelques mois, plusieurs scandales de jeunes hommes noirs sans arme tués par les forces de l'ordre dans des circonstances troubles. En 2013, un jury acquitte le neighborhood watch volunteer qui a tué en Floride Trayvon Martin, 17 ans. En 2014, Eric Garner, 43 ans, est étouffé à mort par les policiers de New York qui l'arrêtaient pour vente de cigarettes à l'unité. Une vidéo amateur filme sa mort, après qu'il répète en suppliant: «je ne peux pas respirer». Michael Brown, 19 ans, est tué par un policier à Ferguson (Missouri). Akai Gurley, 28 ans, est tué par un policier dans une cage d'escalier de Brooklyn, dans la pénombre, alors qu'il rendait visite à une amie. Tamir Rice, 12 ans, est tué dans l'Ohio par un policier qui a confondu le jouet de l'enfant avec une arme véritable. En 2015, Walter Scott, 50 ans, est tué par un policier alors qu'il s'enfuyait après avoir été arrêté pour un défaut de feu de stop sur sa voiture. Une vidéo amateur montre le policier lui tirant dans le dos, à moins de dix mètres de distance. Freddie Gray, 25 ans, meurt des suites de ses blessures à la colonne vertébrale, vraisemblablement infligées par les

\footnotetext{
${ }^{1}$ Useem, B. (1998). Breakdown theories of collective action. Annual Review of Sociology, 24 : 215-238.

${ }^{2}$ Olzak, S., Shanahan, S., \& McEneaney, E. H. (1996). Poverty, segregation, and race riots: 1960 to 1993. American Sociological Review, 61 (4): 590-613.

${ }^{3}$ Portes, A., \& Stepick, A. (1993). City on the edge: The transformation of Miami. Univ of California Press.

${ }^{4}$ Bergesen, A., \& Herman, M. (1998). Immigration, race, and riot: The 1992 Los Angeles uprising. American Sociological Review, 39-54.

${ }^{5}$ Katz, M. B. (2008). Why don't American cities burn very often?. Journal of Urban History, 34(2), 185-208.
} 
policiers lors de son transport dans un camion de police. Je ne cite que les affaires qui ont eu un grand écho dans les médias ; plus de 1000 personnes sont abattues par la police tous les ans aux Etats-Unis.

Les morts de Michael Brown et Freddie Gray déclenchent les émeutes de Ferguson (20142015, une dizaine de blessés) et Baltimore (2015, plus de 100 policiers blessés).

\section{La police et les émeutes}

Aux Etats-Unis comme en France, les émeutes des trente dernières années ont quasiment toutes pour déclencheur un incident avec la police. C'est moins vrai pour les émeutes des années 1960, mais les émeutes les plus graves (Los Angeles 1965, Detroit 1967, Newark 1967) sont également déclenchées par un incident avec la police (celles de 1968 font suite à l'assassinat de Martin Luther King). Pour la sociologie spilermanienne, la question de la police est de l'ordre de la pure contingence. Pour Spilerman, ces incidents déclencheurs n'ont aucun intérêt, puisqu'ils sont routiniers et présents partout, et qu'il n'y a pas d'émeutes partout. Donc, les émeutes ont des causes profondes (underlying causes) à chercher dans les variables qui ont «la plus haute signification sociologique » (je cite Spilerman), c'est-à-dire les variables socio-économiques et démographiques.

Dans ce raisonnement, il y a une forme de cécité au réel qui chagrine. Si les émeutes sont massivement causées par le comportement de la police, et que ce comportement est routinier dans les ghettos noirs, alors le comportement de la police doit être étudié comme une variable structurelle, et non comme une contingence.

Du reste, pendant les émeutes des années 1960, certains sociologues se sont penchés sur la question policière. Herbert Gans a souligné le problème qu'il y a à confier le maintien de l'ordre à des policiers blancs, issus des classes populaires, "seulement un cran au-dessus du [Noir] en statut social, parfois en compétition avec lui pour les emplois, et qui sont souvent les plus ardemment anti-[Noirs] ${ }^{1} \gg$. Anthony Oberschall, future figure de la sociologie des mouvements sociaux, analyse l'émeute de Los Angeles de 1965 (34 morts). Il met en avant le rôle joué par le contexte de violences policières, ainsi que le passage au policing fondé sur les patrouilles en voiture (et non à pied), dont l'indicateur de performance est le temps de réponse aux appels d'urgence. Oberschall analyse ce changement fondamental dans les tactiques policières comme une cause de la perte de légitimité de la police auprès des populations les plus défavorisées - une analyse qui anticipe les travaux sur le community policing («police de proximité ») à la fin des années $1970^{2}$. Toutes ces réflexions sur les relations policepopulation sont d'ailleurs la conséquence des émeutes et de la peur d'une insurrection généralisée des Noirs.

\section{Conclusion}

La gravité des émeutes américaines est sans commune mesure avec les émeutes dans les banlieues françaises depuis les années 1970 (deux morts en 2005). On peut néanmoins essayer de tirer des leçons pour la sociologie française des émeutes. Il y a dans la littérature française une interrogation insistante sur les griefs supposés des émeutiers. Régis Cortesero et Eric Marlière analysent la sociologie française des émeutes post-2005 comme «orpheline du mouvement ouvrier », animée par la «nostalgie d'un grand acteur populaire », sans prise sur ces émeutes dont on n'arrive pas a savoir si elle sont « ethniques », « urbaines » ou « sociales

\footnotetext{
${ }^{1}$ Gans, op. cit., p. 45

2 Oberschall, A. (1968). The Los Angeles riot of August 1965. Social Problems, 15(3), 322-341.
} 
(économiques) ${ }^{1}$. L'article ravageur de Frédéric Ocqueteau au lendemain des émeutes de 2005 avait montré comment les griefs identifiés dans les différents travaux reflétaient fidèlement les opinions politiques et les centres d'intérêts scientifiques de leurs auteurs respectifs. Comme les émeutiers n'articulent pas de revendications, les sociologues se chargent de parler pour eux ${ }^{2}$. Il ne faudrait cependant pas jeter le bébé avec l'eau du bain : les événements de Ferguson et Baltimore montre le côté un peu absurde de la démarche qui consiste à traiter les émeutes comme de simples pillages festifs. Dans leurs déclinaisons françaises et américaines, les émeutes ont manifestement quelque chose à voir avec la relégation urbaine, la vulnérabilité socio-économique et la domination raciale. En outre, il me semble erroné de réduire la question de la police à une question de circonstances contingentes. Clairement, les événements de Ferguson et Baltimore ont quelque chose à voir avec l'intensité choquante de la répression policière dans les ghettos noirs, et fournissent des arguments à tous ceux qui s'inquiètent de l'incarcération de masse et de la militarisation de la police.

\section{Remerciements}

Je remercie Renaud Epstein et Guillaume Roux pour leur utile lecture critique.

\footnotetext{
1 Cortéséro, R., \& Marlière, É. (2015). L’émeute est-elle une forme d'expression politique?. Agora débats/jeunesses, (2), 57-77.

${ }^{2}$ Ocqueteau, F. (2007). Les émeutes urbaines de l'automne 2005: cadres d'analyse et points aveugles de la sociologie française. Sociologie du travail, 49(4), 531-543.
} 